\title{
Sphingosine kinase I as an anticancer therapeutic target
}

\author{
This article was published in the following Dove Press journal: \\ Drug Design, Development and Therapy \\ 23 June 2015 \\ Number of times this article has been viewed
}

\author{
Ying Gao',* \\ Fei $\mathrm{Gao}^{2, *}$ \\ Kan $\mathrm{Chen}^{3, *}$ \\ Mei-li Tian' \\ Dong-li Zhao' \\ 'Department of Radiotherapy \\ Oncology, First Affiliated Hospital \\ of Xi'an Jiaotong University, \\ ${ }^{2}$ Department of Neurology, First \\ Affiliated Hospital of Xi'an Medica \\ University, Xi'an, ${ }^{3}$ School of Life \\ Sciences, Zhejiang Sci-Tech University, \\ Hangzhou, People's Republic of China \\ *These authors contributed equally \\ to this work
}

\begin{abstract}
The development of chemotherapeutic resistance is a major challenge in oncology. Elevated sphingosine kinase 1 (SK1) levels is predictive of a poor prognosis, and SK1 overexpression may confer resistance to chemotherapeutics. The SK/sphingosine-1-phosphate (S1P)/sphingosine-1-phosphate receptor (S1PR) signaling pathway has been implicated in the progression of various cancers and in chemotherapeutic drug resistance. Therefore, SK1 may represent an important target for cancer therapy. Targeting the SK/S1P/S1PR signaling pathway may be an effective anticancer therapeutic strategy, particularly in the context of overcoming drug resistance. This review summarizes our current understanding of the role of SK/S1P/S1PR signaling in cancer and development of SK1 inhibitors.
\end{abstract}

Keywords: sphingosine kinase 1, S1P, S1PR, inhibitors, cancer, therapy

\section{Introduction}

Cancer is a prominent killer. Oncologists rely heavily on chemotherapy in the fight against cancer. However, some patients exhibit chemotherapeutic resistance, which makes chemotherapy ineffective, resulting in unrestrained metastasis and ultimately, death. Thus, there is a need to study the mechanisms underlying the development of chemotherapeutic resistance so that effective therapies can be developed for patients who show signs of chemotherapeutic resistance.

\section{SKI and cancer transformation}

Sphingosine kinase 1 (SK1) has well-established prosurvival functions in cancer cells. Indeed, the transformation potential of SK1 suggests that it may function as an oncogene. ${ }^{1}$ The SK1 enzyme is activated by an oncogenic form of eukaryotic elongation factor 1A lacking a GDP-GTP binding domain, called PTI-1, and SK1 activation is required for PTI-1-induced neoplastic transformation. ${ }^{2}$

SK1 activity is increased when it is phosphorylated on its Ser225 residue by ERK-2. ${ }^{3}$ Furthermore, phosphorylation of SK1 at Ser225 is important for its expression in the plasma membrane, and targeting of SK1 to the plasma membrane increases SK1's capacity to promote transformation. ${ }^{4}$ Indeed, artificial targeting of tagged S225A SK1 mutant protein to the plasma membrane promotes transformation. CIB1, aka calmyrin, has also been shown to facilitate $S K 1$ translocation to the plasma membrane through its involvement in a calcium-myristoyl switch. ${ }^{5}$ The Asn89 and Thr54 residues of $S K 1$ are also important contributors to SK1's selective affinity to the plasma membrane. Specific interaction with the phosphatidyl serines in these residues makes sphingosine available for generation of sphingosine-1-phosphate (S1P) by SK1. S1P released into the extracellular milieu engages with sphingosine-1-phosphate receptors
Correspondence: Ying Gao; Dong-li Zhao Department of Radiotherapy Oncology, First Affiliated Hospital of Xi'an Jiaotong University, 277 Yan Ta West Road, Xi'an city, Xi'an 71006I, People's Republic of China Tel +8602985324029 Email togaoying@163.com; jdyfy223@163.com 
(S1PRs) to induce prosurvival functions. ${ }^{6}$ There are five $\mathrm{G}$ protein-coupled receptors (S1PR1-5), and these are activated in an autocrine/paracrine manner.

Researchers have examined the potential role of $S K 1$ in regulating neoplastic transformation in $S K 1$-transfected NIH3T3 fibroblasts and found that $S K 1$ transfection increases the transformation rate of these fibroblasts into fibrosarcoma cells. ${ }^{4}$ These findings are consistent with the notion that SK1 supports cancer transformation and tumor cell survival. ${ }^{1}$ SK1 is also required for Ras-mediated cell transformation. The messenger (m)RNA and protein expression levels of SK1 are two- to eightfold greater in various cancer tissues (eg, breast, lung, ovarian, stomach, and colon cancers) than in noncarcinoma control tissues. Moreover, growing evidence suggests that increased expression of $S K 1$ is associated with enhanced metastasis, decreased survival, and poor prognoses, ${ }^{7-9}$ suggesting that SK1 may be useful as a biomarker of prognosis. ${ }^{8,9}$ Moreover, small interfering (si) RNA-mediated downregulation of SK1 has been reported to reduce migration of breast cancer cells, implicating SK1 as a potential therapeutic target. ${ }^{10,11}$

\section{SKI's role in cancer progression, metastasis, and apoptosis}

$\mathrm{S} 1 \mathrm{P}$, the product of SK1, binds tumor necrosis factor receptor-associated factor, an E3 ubiquitin ligase that modulates tumor necrosis factor- $\alpha$-induced activation of NF- $\kappa \mathrm{B}$ signaling and induces K63-mediated polyubiquitination of receptor-interacting protein 1 , leading to $\mathrm{I} \kappa \mathrm{B}$ degradation. ${ }^{12}$ $\mathrm{S} 1 \mathrm{P}$ regulation of NF- $\kappa \mathrm{B}$ signaling is consistent with the notion that SK1/S1P may be involved in cancer progression.

Growing evidence points to a role of SK1-derived systemic $\mathrm{S} 1 \mathrm{P}$ in mediating tumor metastasis. A recent finding suggests that serum S1P (not tumor S1P) is important for metastasis to the lungs. In addition, the anti-S1P antibody Sphingomab ${ }^{\mathrm{TM}}$ has been reported to suppress lung metastasis by neutralizing both circulating and systemic S1P, and upregulating breast cancer metastasis suppressor 1 levels. Expression of breast cancer metastasis suppressor 1 is upregulated in cancer cells under conditions of systemic SK1 deficiency, through activation of the S1PR2 signaling pathway. ${ }^{13,14}$ These findings suggest that the SK1 signaling pathway blockade may represent a promising strategy for inhibiting metastasis.

S1P stimulates fibrosarcoma cell migration via activation of GTPases (eg, RAC1 and CDC42). ${ }^{15,16}$ Interestingly, S1P facilitates the migration of S1PR3-expressing gastric cancer cells but suppresses the motility of cell lines with predominant SIPR2 expression. ${ }^{17}$ Meanwhile S1PR1 and S1PR3 have been implicated in ovarian cancer cell invasion, through activation of calcium mobilization and phospholipase C. ${ }^{18}$ S1P binding to S1PR3 was shown to stimulate the accumulation of phosphorylated ERK-1/2 into membrane ruffles/ lamellipodia and promote the migration of MCF-7 breast cancer cells. ${ }^{19}$ Elimination of SK1 resulted in reduced S1PR3 expression and attenuated ERK-1/2 pathway stimulation, leading to a lesser cancer cell migration. These findings suggest that regulation of S1PR3 expression, in particular, may help control metastasis.

SK1 has also been shown to exert antiapoptotic effects through the BAD-BCL2 pathway, wherein mitochondrion-to-cytoplasm translocation of Smac/DIABLO and cytochrome $c$, which is important for apoptosis, is inhibited. ${ }^{20}$ Additionally, SK1 has been reported to protect against the apoptotic effects of sphingosine/ceramide via a delayed BCL2-independent pathway. ${ }^{21}$ Thus, expressing high levels of SK1 appears to shield cancer cells from apoptosis.

\section{SKI and cancer prognosis}

Elevation of SK1 in tumors suggests a potential prognostic application. Increases in SK1 protein and mRNA expression accompany breast cancer progression. By way of an apparent negative feedback process, HER2-induced increases in SK1 levels result in reduced HER2 expression in estrogen receptor (ER)-positive breast cancer cells, thereby preventing S1P-induced migration of these cells. ${ }^{19}$ Meanwhile, high SK1 expression levels in ER-negative breast cancer tumors have been associated with tamoxifen resistance, a higher chance of metastasis, and reduced survival. ${ }^{19,22,23}$ Furthermore, patients with ER-positive breast cancer who had high ERK-1/2 and cytoplasmic SK1 levels were found to experience recurrence 10.5 years earlier, on average, than patients with low levels. ${ }^{22}$ Therefore, clinical phenotype is an important consideration for the clinical application of SK1 inhibitors.

High $S K 1$ expression has also been associated with poor prognosis in patients with a glioblastoma multiforme (aka grade 4 astrocytoma) diagnosis, and SK1-knockdown can reduce proliferation of glioblastoma cells. ${ }^{24}$ Furthermore, SK is necessary for glioma cell invasion and basal activity of the urokinase plasminogen activator system in glioblastoma multiforme. ${ }^{25}$ High expression of SK1 also correlates with higher grade and shorter survival time in non-small cell lung cancer, gastric cancer, non-Hodgkin lymphoma, salivary gland carcinoma, esophageal carcinoma, astrocytoma, and head and neck squamous cell carcinoma. ${ }^{26-32}$ Interestingly, exogenous SK1 has been reported to enhance tumor cell invasiveness despite the fact that it is already overexpressed in many cancers. 
Importantly, the relationship between SK1/S1P levels and cancer progression may differ between different forms of cancer. Although Gleason score and treatment failure rate have been found to correlate with tumoral SK1 activity in prostate cancer, patients with prostate cancer were found to have lower circulating levels of S1P in their erythrocytes than healthy controls. ${ }^{33,34}$ Moreover, decreased circulating S1P has been implicated as an early marker of cancer progression to hormonal unresponsiveness, and circulating S1P levels have been shown to correlate inversely with prostate-specific antigen levels and lymph node metastasis. ${ }^{34}$

\section{Elevated SKI and chemotherapeutic resistance}

Overexpression of SK1 may confer the development of resistance to chemotherapeutics, whereas disruption of SK1/ S1P signaling may restore or improve sensitivity. The multidrug resistant phenotype is associated with an overexpression of P-glycoprotein 1 (aka multidrug resistance protein 1) in tumor cells. Elevated SK1 has been associated with pancreatic cancer cell resistance to gemcitabine and chronic myeloid leukemia (CML) resistance to imatinib. ${ }^{35,36}$ Conversely, SK1 inhibition can sensitize pancreatic cancer and CML cells to the proapoptotic effects of gemcitabine and imatinib, respectively, apparently by increasing the ceramide/S1P ratio and thereby enabling $\mathrm{C} 18$-ceramide-dependent apoptosis to proceed. ${ }^{35-37}$ Thus, the relationship between the SK1/S1P pathway and sensitivity of cancer cells to chemotherapeutics may be due to a direct relation between bioactive sphingolipid levels and drug resistance. Indeed, SK1 has been shown to alter imatinib-induced apoptosis in primary CML cells, ${ }^{37}$ and resistance to etoposide- or doxorubicin-induced apoptosis can be restored with an SK inhibitor (eg, F-12509a), supporting the notion that chemotherapeutic resistance may be the result of reduced ceramide accumulation and sustained SK1 activity. ${ }^{38}$ Attenuation of SK1/S1P signaling can also improve the growth-inhibitory effects of nilotinib. ${ }^{39}$ SK1 elimination or inhibition has been shown to stimulate apoptosis, whereas overexpression of SK1 was shown to reduce caspase activity and reduce apoptosis in imatinib-sensitive cells. ${ }^{40}$ Likewise, daunorubicin sensitivity has been associated with low S1P and high ceramide levels, and it can be restored in daunorubicin-resistant cells by inhibition of SK1 activity or siRNA repression of $S K 1$ expression. ${ }^{40}$ Consistent with these findings, metastatic colon cancer biopsies have shown higher SK1 expression than nonmetastatic specimens, whereas SK1 expression is negligible to nondetectable in normal colon mucosa. ${ }^{41}$ Importantly, given that colon cancer is considered to be a COX2-regulated cancer, knockdown of $S K 1$ expression with siRNA reduces expression of COX2 and production of the $\mathrm{COX} 2$ product prostaglandin $\mathrm{E} 2$ in colon cancer cells. ${ }^{41}$ PC3 (androgen-insensitive) prostate cancer cells been shown to have upregulated SK1 levels and S1PR1/S1PR3 signaling, and are resistant to camptothecin treatment. However, these cells were shown to become sensitive to camptothecin following inhibition of SK. ${ }^{42}$ Likewise, increased SK1 activity also appears to be related to daunorubicin resistance in leukemia cells, cisplatin resistance in lung cancer cells, oxaliplatin resistance in colon cancer cells, and $N$-(4-hydroxyphenyl)retinamide resistance in ovarian carcinoma cells, as well as to docetaxel, doxorubicin, and tamoxifen resistance in breast cancer cells. ${ }^{37,43-46}$ Accordingly, knockdown or inhibition of $S K 1$ has been reported to enhance drug efficacy in leukemia cells, ovarian cancer, and colon cancer. ${ }^{38,44,45}$ Hence, together, the aforementioned findings suggest strongly that $S K 1$ plays a biologically significant role in chemotherapeutic drug resistance.

Different S1PRs appear to play the dominant the role in SK1/S1P-related chemoresistance across different cancers. S1PR2 mRNA was found to be elevated in nephroblastoma tissue relative to levels in healthy kidney tissue. ${ }^{47}$ Additionally, in nephroblastoma cells, it was shown that $S 1 P R 2$ overexpression resulted in increased $\mathrm{mRNA}$ and protein expression of $C O X 2$ and, correspondingly, increased synthesis of its product prostaglandin E2, and these effects could be blocked by S1PR2 antagonism, demonstrating that S1PR2 signaling is a strong driver of renal cancer progression. ${ }^{47}$ Meanwhile, cytoplasmic expression levels of S1PR1 and S1PR3 in ER-positive breast cancer tumors associate negatively with patient survival. ${ }^{22}$ The protective influence of SK1 on prostate cancer cells has been shown to involve S1PR2/3 receptors, whereas chemoresistance can be attenuated with FTY720, a sphingosine analog that inhibits S1PR signaling and induces proteasome-mediated degradation of SK $1 .{ }^{48}$

\section{SKI/S IPR targeting in anticancer therapeutics}

Oncological targeting of SK1/S1P is attractive due to the proapoptotic and antiproliferative potential of SK1 inhibition. Various SK1/S1P and/or S1PR agents have been developed, as elaborated below.

\section{Pan-SK inhibitors}

SK inhibition has been shown to reduce the viability of glioblastoma multiforme cells, neuroblastoma cells, several types of leukemia cells, and various solid tumor cell lines. ${ }^{49-53}$ 
Importantly, SK inhibitors can, not only inhibit growth of temozolomide-resistant ${ }^{49}$ and camptothecin-resistant cancer cells $^{54}$ but also, can reduce proliferation and induce apoptosis in multidrug-resistant tumor cells, ${ }^{7}$ suggesting they may be able to convert chemotherapeutic-resistant tumors into chemosensitive tumors. $N, N$-dimethyl sphingosine (DMS) and L-threo-dihydrosphingosine (Safingol) are competitive SK inhibitors that have inhibitory influences on protein kinase $\mathrm{C}$ and ceramide kinase; they activate sphingosinemediated targets, such as casein kinase 2 and PI3K. ${ }^{55,56}$ DMS, in particular, has been shown to suppress the growth of multiple cancer cell lines ${ }^{57}$ Reported off-target effects of DMS include hemolysis and hepatotoxicity. ${ }^{58}$

\section{Selective SK inhibitors}

With the aim of minimizing the secondary effects of chemotherapy, researchers have sought and developed agents with more selective effects. The selective SK1 inhibitors SK1-I and SK1-II have each been shown to induce apoptosis in T-cell large granular lymphocyte leukemia cells but not normal cells. ${ }^{53}$ Likewise, although SK1-I potently induces apoptosis in leukemic cells, normal leukocytes are relatively spared. ${ }^{52}$ SK1-I has been shown to have efficacy against xenograft glioblastomas as well as orthotopic or acute myeloid leukemia xenograft tumors. ${ }^{52,59}$ Additionally, SK1-I has been shown to decrease serum S1P levels, promote cancer cell apoptosis, and reduce lymph node and lung metastasis in a murine breast cancer model. ${ }^{14}$ Combining SK1-I with a proteasome inhibitor has been reported to yield synergistic antigrowth and proapoptotic effects in imatinib-resistant leukemia cells, and these effects have been associated with downregulation of Mcl-1 and BCR/ABL. ${ }^{60}$ Meanwhile, SK1-II has been shown to induce proteasomal degradation of SK1 in androgen-sensitive prostate cancer, breast cancer, and human pulmonary artery smooth muscle cells. ${ }^{61}$

There is great interest in the anticancer potential of the synthetic sphingosine analog FTY720 (2-amino-2[2-(4-octylphenyl)]-1,3-propanediolhydrochloride), aka fingolimod. FTY720 is a competitive sphingosine inhibitor of SK1, and its analog (S)-FTY720 vinylphosphonate is a noncompetitive inhibitor of SK1. FTY720 and (S)-FTY720 vinylphosphonate have been reported to stimulate relocalization of actin away from the lamellipodia of breast cancer cells, suggesting these inhibitors' possible application for prevention of tumor metastasis. ${ }^{19,62}$

Although the mechanism of FTY720's anticancer effects has not been clarified, evidence suggests that it may work through both caspase-dependent and caspase-independent apoptotic pathways. FTY720 has been found to inhibit breast and colon cancer cell lines through S1PR-independent effects. ${ }^{20}$ Binding of FTY720-phosphate on T-lymphocytes to S1P1 was shown to induce S1P1 downregulation and lymphopenia. ${ }^{63}$ Both FTY720 and its analog (S)-FTY720 vinylphosphonate were shown to induce proteasomal degradation of SK1 and apoptosis in prostate and breast cancer cells. ${ }^{48}$ They also prevented S1P-stimulated actin rearrangement in MCF-7 cells. ${ }^{62}$ FTY720 has been reported to increase prostate cancer sensitivity to radiation, and to reduce tumor growth and metastasis. ${ }^{64}$ Meanwhile, SEW2871 (a S1PR1-specific antagonist) has been shown to suppress angiogenesis, indicating a receptor-dependent function. ${ }^{64,65}$ Other S1PR1 and S1PR1/3 receptor antagonists, such as VPC2309, VPC4416, W146, and VPC25239, have shown promising results in situ. ${ }^{66,67}$

FTY720 is currently Food and Drug Administration (FDA)-approved for use as an immunosuppressant in patients with multiple sclerosis. ${ }^{63}$ However, a great deal more research is required to delineate the receptor-dependent and -independent functions of FTY-720 in the context of its reported inhibitory effects on cancer cell proliferation. Synthetic SK1-specific inhibitors, such as 9ab, 6ag, and 12aa, have shown promising effects in vitro, but their efficacy has yet to be validated in vivo. ${ }^{68}$

\section{Anti-SIP antibodies}

Anti-S1P monoclonal antibodies that specifically neutralize and target S1P have been reported to be effective against breast MDA-MB-231, lung A549, ovarian SKOV3, and melanoma F16/B10 cancer models in vivo and in situ. Anti-S1P antibodies, which function somewhat like a molecular sponge to neutralize S1P signaling, have been reported to favor tumor regression and inhibit lung metastasis in xenograft and allograft models. Sphingomab (LT1002) and its humanized form (LT1009), not only neutralize VEGFand bFGF-induced angiogenesis but also, block $\mathrm{S} 1$-induced endothelial cell tube migration and formation in numerous assays. $^{69}$

\section{Conclusion}

An accumulation of evidence indicates that the SK/S1P/S1PR signaling pathway plays a crucial role in various cancers and in sphingolipid-mediated drug resistance. Elevated expression of SK1 leads to an oncogenic phenotype, through SK1 effects on S1P production, and sphingosine and ceramide accumulation. Conversely, administration of SK1 inhibitors induces proteasomal degradation. Therefore, SK1 represents 
a particularly interesting target for cancer therapy. Targeting the SK/S1P/S1PR signaling pathway may be an effective anticancer therapeutic strategy, particularly for overcoming drug resistance.

\section{Acknowledgments}

This study was supported by The National Natural Science Foundation of China (grant number 81301937) and by the International Cooperation Foundation of Shaanxi Province of China (grant number 2013KW-27-03).

\section{Disclosure}

The authors report no conflicts of interest in this work. This paper has not been published previously.

\section{References}

1. Xia P, Gamble JR, Wang L, et al. An oncogenic role of sphingosine kinase. Curr Biol. 2000;10(23):1527-1530.

2. Leclercq TM, Moretti PA, Pitson SM. Guanine nucleotides regulate sphingosine kinase 1 activation by eukaryotic elongation factor $1 \mathrm{~A}$ and provide a mechanism for eEF1A-associated oncogenesis. Oncogene. 2011;30(3):372-378.

3. Selvam SP, Ogretmen B. Sphingosine kinase/sphingosine 1-phosphate signaling in cancer therapeutics and drug resistance. Handb Exp Pharmacol. 2013;(216):3-27.

4. Pitson SM, Xia P, Leclercq TM, et al. Phosphorylation-dependent translocation of sphingosine kinase to the plasma membrane drives its oncogenic signalling. J Exp Med. 2005;201(1):49-54.

5. Jarman KE, Moretti PA, Zebol JR, Pitson SM. Translocation of sphingosine kinase 1 to the plasma membrane is mediated by calcium- and integrin-binding protein 1. J Biol Chem. 2010;285(1):483-492.

6. Stahelin RV, Hwang JH, Kim JH, et al. The mechanism of membrane targeting of human sphingosine kinase 1.J Biol Chem. 2005;280(52) 43030-43038.

7. Pyne NJ, Pyne S. Sphingosine 1-phosphate and cancer. Nat Rev Cancer. 2010;10(7):489-503

8. Zhang Y, Wang Y, Wan Z, Liu S, Cao Y, Zeng Z. Sphingosine kinase 1 and cancer: a systematic review and meta-analysis. PLoS One. 2014;9(2): e90362.

9. Pyne S, Edwards J, Ohotski J, Pyne NJ. Sphingosine 1-phosphate receptors and sphingosine kinase 1: novel biomarkers for clinical prognosis in breast, prostate, and hematological cancers. Front Oncol. 2012;2:168.

10. Döll F, Pfeilschifter J, Huwiler A. Prolactin upregulates sphingosine kinase-1 expression and activity in the human breast cancer cell line MCF7 and triggers enhanced proliferation and migration. Endocr Relat Cancer. 2007;14(2):325-335.

11. Sarkar S, Maceyka M, Hait NC, et al. Sphingosine kinase 1 is required for migration, proliferation and survival of MCF-7 human breast cancer cells. FEBS Lett. 2005;579(24):5313-5317.

12. Alvarez SE, Harikumar KB, Hait NC, et al. Sphingosine-1-phosphate is a missing cofactor for the E3 ubiquitin ligase TRAF2. Nature. 2010; 465(7301):1084-1088.

13. Ponnusamy S, Selvam SP, Mehrotra S, et al. Communication between host organism and cancer cells is transduced by systemic sphingosine kinase 1/sphingosine 1-phosphate signalling to regulate tumour metastasis. EMBO Mol Med. 2012;4(8):761-775.

14. Nagahashi M, Ramachandran S, Kim EY, et al. Sphingosine-1-phosphate produced by sphingosine kinase 1 promotes breast cancer progression by stimulating angiogenesis and lymphangiogenesis. Cancer Res. 2012;72(3):726-735.
15. Fisher KE, Pop A, Koh W, Anthis NJ, Saunders WB, Davis GE. Tumor cell invasion of collagen matrices requires coordinate lipid agonist-induced G-protein and membrane-type matrix metalloproteinase-1-dependent signaling. Mol Cancer. 2006;5:69.

16. Nyalendo C, Michaud M, Beaulieu E, et al. Src-dependent phosphorylation of membrane type I matrix metalloproteinase on cytoplasmic tyrosine 573: role in endothelial and tumor cell migration. J Biol Chem. 2007;282(21):15690-15699.

17. Yamashita H, Kitayama J, Shida D, et al. Sphingosine 1-phosphate receptor expression profile in human gastric cancer cells: differential regulation on the migration and proliferation. J Surg Res. 2006;130(1): 80-87.

18. Park KS, Kim MK, Lee HY, et al. S1P stimulates chemotactic migration and invasion in OVCAR3 ovarian cancer cells. Biochem Biophys Res Commun. 2007;356(1):239-244.

19. Long JS, Edwards J, Watson C, et al. Sphingosine kinase 1 induces tolerance to human epidermal growth factor receptor 2 and prevents formation of a migratory phenotype in response to sphingosine 1-phosphate in estrogen receptor-positive breast cancer cells. Mol Cell Biol.2010;30(15): $3827-3841$

20. Pyne NJ, Tonelli F, Lim KG, Long J, Edwards J, Pyne S. Targeting sphingosine kinase 1 in cancer. Adv Biol Regul. 2012;52(1):31-38.

21. Leroux ME, Auzenne E, Evans R, et al. Sphingolipids and the sphingosine kinase inhibitor, SKI II, induce BCL-2-independent apoptosis in human prostatic adenocarcinoma cells. Prostate. 2007;67(15): 1699-1717.

22. Watson C, Long JS, Orange C, et al. High expression of sphingosine 1-phosphate receptors, S1P1 and S1P3, sphingosine kinase 1, and extracellular signal-regulated kinase-1/2 is associated with development of tamoxifen resistance in estrogen receptor-positive breast cancer patients. Am J Pathol. 2010;177(5):2205-2215.

23. Ruckhäberle E, Rody A, Engels K, et al. Microarray analysis of altered sphingolipid metabolism reveals prognostic significance of sphingosine kinase 1 in breast cancer. Breast Cancer Res Treat. 2008;112(1): $41-52$.

24. Van Brocklyn JR, Jackson CA, Pearl DK, Kotur MS, Snyder PJ, Prior TW. Sphingosine kinase-1 expression correlates with poor survival of patients with glioblastoma multiforme: roles of sphingosine kinase isoforms in growth of glioblastoma cell lines. J Neuropathol Exp Neurol. 2005; 64(8):695-705.

25. Young N, Pearl DK, Van Brocklyn JR. Sphingosine-1-phosphate regulates glioblastoma cell invasiveness through the urokinase plasminogen activator system and CCN1/Cyr61. Mol Cancer Res. 2009;7(1): 23-32.

26. Song L, Xiong H, Li J, et al. Sphingosine kinase-1 enhances resistance to apoptosis through activation of PI3K/Akt/NF- $\mathrm{KB}$ pathway in human non-small cell lung cancer. Clin Cancer Res. 2011;17(7):1839-1849.

27. Li T, Chen L. [Alphastatin inhibits tumor angiogenesis in nude mice bearing human gastric cancer xenografts]. Zhonghua Wei Chang Wai Ke Za Zhi. 2009;12(1):61-64. Chinese.

28. Bayerl MG, Bruggeman RD, Conroy EJ, et al. Sphingosine kinase 1 protein and mRNA are overexpressed in non-Hodgkin lymphomas and are attractive targets for novel pharmacological interventions. Leuk Lymphoma. 2008;49(5):948-954.

29. Liu G, Zheng H, Zhang Z, et al. Overexpression of sphingosine kinase 1 is associated with salivary gland carcinoma progression and might be a novel predictive marker for adjuvant therapy. BMC Cancer. 2010;10: 495.

30. Pan J, Tao YF, Zhou Z, et al. An novel role of sphingosine kinase-1 (SPHK1) in the invasion and metastasis of esophageal carcinoma. J Transl Med. 2011;9:157.

31. Li J, Guan HY, Gong LY, et al. Clinical significance of sphingosine kinase-1 expression in human astrocytomas progression and overall patient survival. Clin Cancer Res. 2008;14(21):6996-7003.

32. Facchinetti MM, Gandini NA, Fermento ME, et al. The expression of sphingosine kinase-1 in head and neck carcinoma. Cells Tissues Organs. 2010;192(5):314-324. 
33. Malavaud B, Pchejetski D, Mazerolles C, et al. Sphingosine kinase-1 activity and expression in human prostate cancer resection specimens. Eur J Cancer. 2010;46(18):3417-3424.

34. Nunes J, Naymark M, Sauer L, et al. Circulating sphingosine-1-phosphate and erythrocyte sphingosine kinase-1 activity as novel biomarkers for early prostate cancer detection. Br J Cancer. 2012;106(5):909-915.

35. Guillermet-Guibert J, Davenne L, Pchejetski D, et al. Targeting the sphingolipid metabolism to defeat pancreatic cancer cell resistance to the chemotherapeutic gemcitabine drug. Mol Cancer Ther. 2009;8(4):809-820.

36. Baran Y, Salas A, Senkal CE, et al. Alterations of ceramide/sphingosine 1-phosphate rheostat involved in the regulation of resistance to imatinibinduced apoptosis in K562 human chronic myeloid leukemia cells. J Biol Chem. 2007;282(15):10922-10934.

37. Bonhoure E, Lauret A, Barnes DJ, et al. Sphingosine kinase-1 is a downstream regulator of imatinib-induced apoptosis in chronic myeloid leukemia cells. Leukemia. 2008;22(5):971-979.

38. Heffernan-Stroud LA, Obeid LM. Sphingosine kinase 1 in cancer. $A d v$ Cancer Res. 2013;117:201-235.

39. Salas A, Ponnusamy S, Senkal CE, et al. Sphingosine kinase-1 and sphingosine 1-phosphate receptor 2 mediate Bcr-Abl1 stability and drug resistance by modulation of protein phosphatase 2A. Blood. 2011; 117(22):5941-5952.

40. Sobue S, Nemoto S, Murakami M, et al. Implications of sphingosine kinase 1 expression level for the cellular sphingolipid rheostat: relevance as a marker for daunorubicin sensitivity of leukemia cells. Int J Hematol. 2008;87(3):266-275.

41. Kawamori T, Osta W, Johnson KR, et al. Sphingosine kinase 1 is upregulated in colon carcinogenesis. FASEB J. 2006;20(2):386-388.

42. Bektas M, Jolly PS, Müller C, Eberle J, Spiegel S, Geilen CC. Sphingosine kinase activity counteracts ceramide-mediated cell death in human melanoma cells: role of Bcl-2 expression. Oncogene. 2005;24(1): 178-187.

43. Min J, Van Veldhoven PP, Zhang L, Hanigan MH, Alexander H, Alexander S. Sphingosine-1-phosphate lyase regulates sensitivity of human cells to select chemotherapy drugs in a p38-dependent manner. Mol Cancer Res. 2005;3(5):287-296.

44. Nemoto S, Nakamura M, Osawa Y, et al. Sphingosine kinase isoforms regulate oxaliplatin sensitivity of human colon cancer cells through ceramide accumulation and Akt activation. J Biol Chem. 2009; 284(16):10422-10432.

45. Illuzzi G, Bernacchioni C, Aureli M, et al. Sphingosine kinase mediates resistance to the synthetic retinoid N-(4-hydroxyphenyl) retinamide in human ovarian cancer cells. J Biol Chem. 2010;285(24): 18594-18602.

46. Sukocheva O, Wang L, Verrier E, Vadas MA, Xia P. Restoring endocrine response in breast cancer cells by inhibition of the sphingosine kinase-1 signaling pathway. Endocrinology. 2009;150(10):4484-4492.

47. Li MH, Sanchez T, Milne GL, Morrow JD, Hla T, Ferrer F. S1P/S1P2 signaling induces cyclooxygenase-2 expression in Wilms tumor. JUrol. 2009;181(3):1347-1352.

48. Tonelli F, Lim KG, Loveridge C, et al. FTY720 and (S)-FTY720 vinylphosphonate inhibit sphingosine kinase 1 and promote its proteasomal degradation in human pulmonary artery smooth muscle, breast cancer and androgen-independent prostate cancer cells. Cell Signal. 2010;22(10):1536-1542.

49. Bektas M, Johnson SP, Poe WE, Bigner DD, Friedman HS. A sphingosine kinase inhibitor induces cell death in temozolomide resistant glioblastoma cells. Cancer Chemother Pharmacol. 2009;64(5): 1053-1058

50. Tavarini S, Colombaioni L, Garcia-Gil M. Sphingomyelinase metabolites control survival and apoptotic death in SH-SY5Y neuroblastoma cells. Neurosci Lett. 2000;285(3):185-188.

51. Cuvillier O, Levade T. Sphingosine 1-phosphate antagonizes apoptosis of human leukemia cells by inhibiting release of cytochrome $\mathrm{c}$ and Smac/DIABLO from mitochondria. Blood. 2001;98(9):2828-2836.
52. Paugh SW, Paugh BS, Rahmani M, et al. A selective sphingosine kinase 1 inhibitor integrates multiple molecular therapeutic targets in human leukemia. Blood. 2008;112(4):1382-1391.

53. Zhang R, Shah MV, Yang J, et al. Network model of survival signaling in large granular lymphocyte leukemia. Proc Natl Acad Sci US A. 2008; 105(42):16308-16313.

54. Akao Y, Banno Y, Nakagawa Y, et al. High expression of sphingosine kinase 1 and S1P receptors in chemotherapy-resistant prostate cancer PC3 cells and their camptothecin-induced up-regulation. Biochem Biophys Res Commun. 2006;342(4):1284-1290.

55. King CC, Zenke FT, Dawson PE, et al. Sphingosine is a novel activator of 3-phosphoinositide-dependent kinase 1. J Biol Chem. 2000; 275(24):18108-18113.

56. Sugiura M, Kono K, Liu H, et al. Ceramide kinase, a novel lipid kinase. Molecular cloning and functional characterization. J Biol Chem. 2002; 277(26):23294-23300.

57. Schwartz GK, Ward D, Saltz L, et al. A pilot clinical/pharmacological study of the protein kinase C-specific inhibitor safingol alone and in combination with doxorubicin. Clin Cancer Res. 1997;3(4): 537-543.

58. Kedderis LB, Bozigian HP, Kleeman JM, et al. Toxicity of the protein kinase $\mathrm{C}$ inhibitor safingol administered alone and in combination with chemotherapeutic agents. Fundam Appl Toxicol. 1995;25(2): 201-217.

59. Kapitonov D, Allegood JC, Mitchell C, et al. Targeting sphingosine kinase 1 inhibits Akt signaling, induces apoptosis, and suppresses growth of human glioblastoma cells and xenografts. Cancer Res. 2009;69(17): 6915-6923.

60. Li QF, Yan J, Zhang K, et al. Bortezomib and sphingosine kinase inhibitor interact synergistically to induces apoptosis in BCR/ABl+ cells sensitive and resistant to STI571 through down-regulation Mcl-1. Biochem Biophys Res Commun. 2011;405(1):31-36.

61. Loveridge C, Tonelli F, Leclercq $\mathrm{T}$, et al. The sphingosine kinase 1 inhibitor 2-(p-hydroxyanilino)-4-(p-chlorophenyl)thiazole induces proteasomal degradation of sphingosine kinase 1 in mammalian cells. J Biol Chem. 2010;285(50):38841-38852.

62. Lim KG, Tonelli F, Li Z, et al. FTY720 analogues as sphingosine kinase 1 inhibitors: enzyme inhibition kinetics, allosterism, proteasomal degradation, and actin rearrangement in MCF-7 breast cancer cells. J Biol Chem. 2011;286(21):18633-18640.

63. Brinkmann V, Billich A, Baumruker T, et al. Fingolimod (FTY720): discovery and development of an oral drug to treat multiple sclerosis. Nat Rev Drug Discov. 2010;9(11):883-897.

64. Pchejetski D, Bohler T, Brizuela L, et al. FTY720 (fingolimod) sensitizes prostate cancer cells to radiotherapy by inhibition of sphingosine kinase-1. Cancer Res. 2010;70(21):8651-8661.

65. Nagaoka Y, Otsuki K, Fujita T, Uesato S. Effects of phosphorylation of immunomodulatory agent FTY720 (fingolimod) on antiproliferative activity against breast and colon cancer cells. Biol Pharm Bull. 2008;31(6):1177-1181.

66. Davis MD, Clemens JJ, Macdonald TL, Lynch KR. Sphingosine 1-phosphate analogs as receptor antagonists. J Biol Chem. 2005;280(11): 9833-9841.

67. Sanna MG, Wang SK, Gonzalez-Cabrera PJ, et al. Enhancement of capillary leakage and restoration of lymphocyte egress by a chiral S1P1 antagonist in vivo. Nat Chem Biol. 2006;2(8):434-441.

68. Xiang Y, Asmussen G, Booker M, et al. Discovery of novel sphingosine kinase 1 inhibitors. Bioorg Med Chem Lett. 2009;19(21):6119-6121.

69. Visentin B, Vekich JA, Sibbald BJ, et al. Validation of an anti-sphingosine-1-phosphate antibody as a potential therapeutic in reducing growth, invasion, and angiogenesis in multiple tumor lineages. Cancer Cell. 2006;9(3):225-238. 


\section{Publish your work in this journal}

Drug Design, Development and Therapy is an international, peerreviewed open-access journal that spans the spectrum of drug design and development through to clinical applications. Clinical outcomes, patient safety, and programs for the development and effective, safe, and sustained use of medicines are a feature of the journal, which has also been accepted for indexing on PubMed Central. The manuscript management system is completely online and includes a very quick and fair peer-review system, which is all easy to use. Visit http://www.dovepress.com/testimonials.php to read real quotes from published authors.

Submit your manuscript here: http://www.dovepress.com/drug-design-development-and-therapy-journal 